www. revistadyo.com

\title{
Costos operativos en el transporte de mercancía por carretera: el caso de los sistemas de construcción ligera en México
}

\author{
Luis David Berrones-Sanz
}

Recibido: 11 de Junio de 2020

https://doi.org/10.37610/dyo.v0i73.589

Aceptado: 12 de Enero de 2021

\section{Resumen}

Para analizar la estructura de costes de los proveedores de transporte por carretera en una empresa dedicada a la manufactura de sistemas de construcción ligera, se aplicó el método del Análisis del Ciclo Vehicular. Sus componentes fueron obtenidos a través del seguimiento de 14,823 embarques y una encuesta a las 52 empresas transportistas. Los resultados muestran que $51.7 \%$ del tiempo los vehículos estuvieron sin movimiento, y con un costo promedio de US $\$ 1.19 / \mathrm{km}$. A pesar de las limitaciones cuando las rutas son dinámicas; el método es fácil de sistematizar, facilita el cálculo prospectivo de las tarifas, y aporta elementos durante la elaboración de programas para mejorar la eficiencia de todos los agentes implicados.

\section{Palabras clave}

Costos de autotransporte; Precios del servicio de transporte; Análisis de Ciclo Vehicular; Tarifas de flete.

\section{Introducción}

El análisis de los costos en el transporte de carga proporciona información relevante sobre el desempeño de las tareas, contribuye en el control de los recursos, y se utiliza para evitar gastos innecesarios, establecer precios, planificar actividades, realizar inversiones $\mathrm{y}$, en general, en cualquier toma de decisiones (Hooper y Murray, 2019). De esta forma, las empresas requieren conocer sus costos para eliminar estructuras no productivas e incrementar su competitividad y eficiencia a largo plazo.

No obstante, a pesar de que se ha desarrollado una extensa literatura en torno al problema del análisis de costos en los sistemas de transporte (Zikopoulos, 2019), en México es común que las empresas dedicadas al traslado de mercancías por carretera carezcan de información confiable sobre sus costos de operación (Berrones-Sanz, 2020). Esto debido a que la estructura empresarial del sector está dominado por los micro y pequeños transportistas que conforman 97.2\% del total de las empresas del país (DGAF, 2018). Este tipo de empresas, la mayoría de tipo hombre-camión (owneroperator), carecen de infraestructura, personal administrativo o recursos tecnológicos y, por tanto, no le dan el seguimiento con la importancia y la precisión que el análisis de costos requiere.

\footnotetext{
\uis David Berrones-Sanz * luis.berrones@uacm.edu.mx

Asimismo, el gran número de empresas transportistas en el autotransporte de carga federal $(152,487)$ genera un fuerte nivel de competencia. Bajo este contexto, las denominadas hombre-camión ofrecen tarifas bajas, muchas veces hasta por debajo de sus costos de operación, a cambio de una serie de externalidades que mantienen la flota vehicular nacional con un promedio de 17 años de antigüedad. Así, bajo un desconocimiento sistemático de la estructura de costos y en un mercado donde las empresas embarcadoras han tomado el control de las tarifas, se ven obligados a ser cada vez más competitivos, reducir sus costos de operación y ofrecer actividades adicionales que agregan valor a sus servicios (como el rastreo satelital o algunos servicios logísticos).

Entonces, en las empresas embarcadoras recae la responsabilidad de realizar esfuerzos para mejorar el desempeño de los proveedores que transportan sus mercancías. No sólo se trata de mejorar las capacidades de sus proveedores para alcanzar competitividad al largo plazo, sino por la responsabilidad social y medio ambiental.

De esta forma, se elaboró el estudio de caso de la distribución de productos terminados para la construcción en México, que utilizan como base los paneles de yeso, conocidos como "sistemas de construcción ligera" debido a que su peso es mucho menor que los ladrillos y los materiales convencionales de construcción. El propósito fue estudiar la estructura de costos de los proveedores que trasladan las mercancías por carretera, para esto, se sistematizó el método del Análisis de Ciclo Vehicular y se utilizó como herramienta de intercambio de información y evaluación comparativa para la gestión de costos de las empresas transportistas. 
El trabajo se organiza de la siguiente manera: se inicia con una revisión de estudios sobre los costos de transporte y diferentes metodologías que se han utilizado para el cálculo de los costos, especialmente en México. Se incluye un apartado sobre los conceptos relacionados a los precios y la forma en que se calculan los costos operativos en el transporte de carga por carretera, y otro donde se tratan los aspectos teóricos del método del análisis del ciclo vehicular. Posteriormente, se muestra la metodología del caso de estudio y la descripción de los agentes implicados en el transporte de los productos de construcción ligera. Le sigue el apartado de resultados, donde se incluye el tiempo de cada una de las actividades de los embarques, junto con los costos y movimientos que se utilizaron para aplica el método del Análisis del Ciclo Vehicular, y un apartado donde se realiza un análisis de sensibilidad, en el que se incluyen los tiempos, los desplazamientos y los costos. Finalmente, se presenta la discusión y las conclusiones.

\section{Revisión de la literatura}

Los estudios sobre estimación de costos en el transporte son abundantes en la literatura internacional. Por ejemplo, Litman (2009) realizó una revisión en la que encontró cerca de cuarenta estudios en los que se incluyen de manera general los costos en el transporte, y diecisiete enfocados al transporte de carga que presentan metodologías para cuantificar los costos sociales y ambientales, y que sirven como base para establecer cuotas carreteras, asignar subsidios o realizar acciones relacionadas a las políticas públicas.

Izadi, Nabipour y Titidezh (2020) investigaron la estimación de costos en el transporte de carga. Después de analizar 55 artículos, proponen un modelo costos de transporte de mercancías por carretera cuya estructura se basa en tres clases: los costos operacionales, que incluye costos fijos y costos variables; el tiempo, compuesto por el valor del tiempo y la calidad en el servicio; y los costos externos, que incluye el ambiente, la congestión y los accidentes.

Las metodologías para la estimación de los costos de transporte se pueden clasificar desde tres enfoques: el contable, el estadístico y el cualitativo (Izadi et al., 2020). En los métodos contables se estiman las cantidades en que los elementos del costo de una unidad de producción contribuyen al costo total (Holguín et al., 2013). El principal método del enfoque contable, que supone una relación lineal entre costos y la producción, es el Costeo Basado en Actividades (ABC por sus siglas en inglés). La principal limitación de esta técnica es la imposibilidad de reemplazar los datos ante cambios de escenarios $\mathrm{y}$, por tanto, para determinar y predecir los precios (Izadi et al., 2020). Por su parte, los métodos estadísticos se dividen en modelos econométricos y los modelos estadísticos que no utilizan teoría económica. Un inconveniente de las técnicas estadísticas es que requieren una cantidad significativamente mayor de datos que las técnicas contables (Holguín et al., 2013). Por su parte, los métodos cualitativos son utilizados para analizar información subjetiva, y se utilizan técnicas como encuestas de preferencia reveladas o declaradas para cuantificar datos que no se pueden medir, como por ejemplo el valor del tiempo, la confiabilidad o la comodidad. El problema de los métodos cualitativos, y la subjetividad en general, es que la estimación del valor depende de las personas y sus ideas individuales (Sequera, 2014).

Aunque las metodologías pueden ser utilizadas para establecer precios, estas no se enfocan en el cálculo de tarifas desde la perspectiva del transportista. Zikopoulos (2019) indica que, aunque el problema de la determinación de precios tiene una diversidad de contextos, la literatura concerniente a la idea de fijar precios para el transporte de mercancías, generalmente, se relaciona con los impactos del transporte en el bienestar social y los esfuerzos de los gobiernos para que los responsables del transporte asuman los costos externos. Asimismo, menciona un grupo de investigaciones en los que se utilizan modelos matemáticos para optimizar el ruteo de vehículos y los horarios de transporte (Schwarze, 2016), o las redes de distribución (Tsao et al., 2016); y que buscan proporcionar un enfoque integrado para determinar precios adecuados en los servicios de transporte.

Otro grupo de estudios está integrado por los que exploran las decisiones de precios de transporte que considera la gestión adecuada de los recursos limitados, y que consideran la determinación del precio de transporte para aumentar la rentabilidad de los proveedores de servicios de transporte (Topaloglu y Powell, 2007; Yi et al., 2007; Dondo y Mendez, 2014).

Es común que la literatura sobre establecimiento de precios en el transporte se enfoque en la gestión de los recursos. No obstante, en cuanto a la estructura de costos en el transporte de mercancías por carretea, el Instituto Americano de Investigación en Transporte (ATRI, por sus siglas en inglés) cada año, desde el 2008, realiza un análisis de los costos operativos para proporcionar datos precisos de costos marginales promedio para la industria del transporte. Específicamente, el estudio tiene el objetivo de proporcionar información sobre los costos directos de los transportistas para ser usados como herramienta de evaluación comparativa de alto nivel para los profesionales de la industria y para proporcionar información confiable para las actividades de planificación de embarques. Se revela que, el costo marginal promedio por milla en 2018 fue de US\$1.821 (US\$1.13 por $\mathrm{km}$ ) para el sector del transporte por carretera. Este fue un aumento del $7.7 \%$ con respecto al año anterior, principalmente ocasionado por el aumento de los precios del combustible diésel, los seguros y la escasez de mano de obra (Hooper y Murray, 2019).

Por su parte, en la Unión Europea también se han realizado estudios en los que se analiza la estructura de 
costos para evaluar la competitividad económica de la zona y, específicamente, se compara con los Estados Unidos (Schade et al., 2006). Este estudio encontró que los costos operativos medios por vehículo-kilómetro, en comparación con los Estados Unidos, eran 11.1\% más altos en los países de Europa Occidental y 54\% menor en los países del Este.

Asimismo, en Latinoamérica hay un estudio en el que se estiman los costos y márgenes de utilidad de una empresa de transporte por carretera para el sector agrícola de Chile (Alvear y Rodríguez, 2006). Aquí se obtuvieron los datos, los precios y la eficiencia estándar, constituidos en $84.2 \%$ por el costo del combustible y en el que, finalmente, se estimó un costo total de US\$2.01 por kilómetro; $79.7 \%$ mayor que el estimado para los Estados Unidos en ese año.

En México, las investigaciones sobre los costos en el transporte de carga por carretera son muy escasos, y la mayoría se concentran en el Instituto Mexicano del Transporte (IMT). Así, Arroyo et al. (2016) muestran información sobre los costos de operación del transporte interurbano, en función del alineamiento geométrico y del estado superficial de las carreteras. Los resultados incluyen consumos y costos unitarios de vehículos de carga pesada que son de US\$0.71 por kilómetro $(\mathrm{km})$ para un tractocamión articulado con semirremolque de dos ejes (T3-S2), US\$0.80/km para uno con un semirremolque de tres ejes (T3-S3), y de US\$1.00 $/ \mathrm{km}$ para uno con doble semirremolque (T3-S2-R4). No obstante, el estudio va enfocado en demostrar la importancia de realizar mayor inversión en la construcción de carreteras más durables, con estructuras estables y pavimentos resistentes, que eviten interrupciones en el tránsito, ofrezcan mayor seguridad y reduzcan los costos de los usuarios.

Por su parte, Moreno (2014) presenta un índice de precios en el transporte de carga por carretera (autotransporte), en el que se presentan los insumos básicos (canasta básica), la composición porcentual del gasto -con 38.5\% para combustibles- el efecto sobre los costos de la inflación y el Índice Nacional de Precios y Cotizaciones. Este enfoque es análogo, a las referencias de costos mínimos para el autotransporte de carga general, que elaboraba la Cámara Nacional del Autotransporte de Carga (CANACAR, 2002).

Más adelante, Jiménez y Jiménez (2016) analizan los costos de operación del autotransporte por carretera y la correlación que existe entre la toneladas, la distancia y los fletes. Utilizan el Sistema de Costos del Autotransporte de Mercancías (SI-CAM), que es una herramienta desarrollada por el Instituto Mexicano del Transporte para calcular los costos de operación, de manera personalizada para cada transportista; y que permite establecer metas de análisis de sensibilidad, para mejorar el desempeño de la flota, y determinar el costo de referencia por ruta (IMT, 2018). Sin embargo, a pesar de que es una herramienta muy potente que facilita a los transportistas conocer y graficar sus costos, exige conocer el total de kilómetros recorridos por un vehículo en un año; dato que, si no se tiene estadística previa y se requiere de manera prospectiva, se puede calcular a través del Análisis del Ciclo Vehicular, método que se utiliza como base para fijar los precios y establecer las tarifas en este estudio.

\subsection{Los costos de operación en el transporte de carga por carretera}

Los costos de operación de una empresa de transporte dependen de las cantidades y los precios de sus factores de producción. En general, se sabe que la utilidad es igual a la diferencia entre el ingreso y los costos totales (Rittenberg y Tregarthen, 2018). Los costos totales (CT) se determinan sumando los Costos Fijos (CF) más los Costos Variables (CV) por output (q) o unidad de producción (Kilómetros, tonelada, tonelada-kilómetro, viaje, servicio, metros cúbicos, entre otros).

Por su parte, los costos fijos son todos aquellos gastos que se tienen que cubrir sin importar el nivel de producción. Incluye los Costos Directos (CD) como los derivados por la depreciación, alquiler u obligaciones de los vehículos -seguros e impuestos vehiculares, entre otros- y; Costos Indirectos (CI) compartidos o comunes, como los gastos generales de administración, incluyendo todos aquellos desembolsos periódicos que se realizan con base en contratos, alquiler de oficinas, pago de servicios, agua, luz, teléfono o salarios del personal administrativo; por lo que, para establecer las tarifas en el transporte por carretera, resulta cómodo dividir los costos indirectos entre el número de vehículos y, así, obtener la proporción $\left(\theta_{\mathrm{i}}\right)$ que corresponde a los costos.

De acuerdo con De Rus et al. (2003) los costos en función de la unidad de producción, para un servicio se calculan con la siguiente formula:

$$
\mathrm{C}\left(\mathrm{q}_{\mathrm{i}}\right)=\mathrm{CD}_{\mathrm{i}}+\theta_{\mathrm{i}} \cdot \mathrm{CI}
$$

Donde $\mathrm{CD}_{\mathrm{i}}$ es el costo directo del servicio i y $\theta_{\mathrm{i}}$ es la porción que corresponde a los costos indirectos (CI) atribuidos al servicio i.

Por su parte, los costos variables son los que dependen del nivel de producción, como el combustible, los neumáticos, el mantenimiento, peajes y los gastos del conductor (incluyendo el salario, si no tiene un ingreso fijo). En otras palabras, se puede decir que los costos fijos son los gastos que se tienen que asumir sin importar si los vehículos realizan o no movimientos o servicios; y los costos variables son los que se generan cuando el vehículo está en movimiento.

De esta forma, el costo total anual se calcula sumando los costos fijos acumulados a lo largo de un año $\left(\mathrm{CF}_{\mathrm{a}}\right)$ por los costos variables en función de la unidad de producción $\left[\mathrm{CV}_{\mathrm{i}}\left(\mathrm{q}_{\mathrm{i}}\right)\right]$, tal y como se observa en la siguiente formula. 


$$
\mathrm{CT}_{\mathrm{a}}=\mathrm{CF}_{\mathrm{a}}+\mathrm{CV}_{\mathrm{i}}\left(\mathrm{q}_{\mathrm{i}}\right)
$$

Para establecer una tarifa de transporte en función de una unidad de producción $\left[\mathrm{F}\left(\mathrm{q}_{\mathrm{i}}\right)\right]$, a los costos se le debe incrementar un porcentaje de utilidad $(\% \mathrm{U})$, de la siguiente manera:

$$
\mathrm{F}\left(\mathrm{q}_{\mathrm{i}}\right)=\left[\mathrm{CF}_{\mathrm{i}}+\mathrm{CV}_{\mathrm{i}}\left(\mathrm{q}_{\mathrm{i}}\right)\right] /(1-\% \mathrm{U})
$$

Donde $\mathrm{CF}_{\mathrm{i}}$ son los costos fijos correspondientes al servicio $\mathrm{i}$, y se obtiene dividiendo los Costos Fijos anuales $\left(\mathrm{CF}_{\mathrm{a}}\right)$ entre el consumo por servicio de acuerdo con la unidad de producción, generalmente dada en distancia, tiempo o número de viajes. Para el caso del transporte, es común utilizar como output la distancia que se recorre; por lo que qa podría ser el total anual de kilómetros que un vehículo recorre.

La utilidad total por año $\left(\mathrm{U}_{\mathrm{t}}\right)$ depende de la cantidad de producción anual $\left(\mathrm{q}_{\mathrm{a}}\right)$, y se calcula restando la suma de los costos fijos por año $\left(\mathrm{CF}_{\mathrm{a}}\right)$ y los costos variables de la producción acumulada $\left[\mathrm{CV}_{\mathrm{i}}\left(\mathrm{q}_{\mathrm{i}}\right) \cdot \mathrm{q}_{\mathrm{n}}\right]$, al producto de los ingresos obtenidos por las tarifas $\left[\mathrm{F}\left(\mathrm{q}_{\mathrm{i}}\right)\right]$ y la producción acumulada en el año $\left(q_{n}\right)$, como se muestra a continuación:

$$
\mathrm{U}_{\mathrm{t}}=\mathrm{F}\left(\mathrm{q}_{\mathrm{i}}\right) \cdot \mathrm{q}_{\mathrm{n}}-\left[C \mathrm{CF}_{\mathrm{a}}+\mathrm{CV}_{\mathrm{i}}\left(\mathrm{q}_{\mathrm{i}}\right) \cdot \mathrm{q}_{\mathrm{n}}\right]
$$

La distinción entre la producción anual esperada $\left(\mathrm{q}_{\mathrm{a}}\right) \mathrm{y}$ producción acumulada en el año $\left(\mathrm{q}_{\mathrm{n}}\right)$ se debe a que no siempre se produce lo que se espera; lo ideal es que ambas cantidades sean iguales $(a=n)$. Sin embargo, dado que no siempre es así, resulta de utilidad conocer en qué punto la producción y sus ingresos son suficientes para cubrir los costos totales en el ciclo anual. Para esto, se calcula el punto de equilibrio $\left(\mathrm{q}_{\mathrm{e}}\right)$, que se obtiene igualando a cero la utilidad total $\left(\mathrm{U}_{\mathrm{t}}=0\right)$ y despejando $\mathrm{q}_{\mathrm{n}}$, que se sustituye por qe de la ecuación 4 , por lo que nos queda:

$$
q_{e}=\frac{C F_{a}}{F\left(q_{i}\right)-C V_{i}\left(q_{i}\right)}
$$

De esta manera, la calidad determinística de las ecuaciones para establecer la tarifa, la utilidad o el punto de equilibrio, dispone cálculos bastante simples. La dificultad para establecer precios en el transporte de carga por carretera se encuentra cuando no se dispone de estadísticas previas en los servicios realizados y se pretende determinar los coeficientes de los costos, sobre todo el correspondiente a los costos fijos, que requiere conocer el total de producción anual (como la distancia recorrida por año).

Si el transportista dispone de datos históricos que permitan calcular el número de kilómetros o el total de viajes que recorre un vehículo por año, es fácil establecer la tarifa para un nuevo servicio de las mismas características. Pero, si las estadísticas no están disponibles y se requiere cotizar un nuevo servicio, los datos que encontramos en la literatura o las estimaciones de otros transportistas pueden no ser adecuadas y generar datos erróneos que incrementen o reduzcan la tarifa.

Las consecuencias negativas que esto significa para el servicio y la competitividad de la empresa incluyen pérdidas económicas por ofrecer servicios por debajo del costo de operación; o la pérdida de clientes por incrementos de tarifas no previstas en la cantidad de producción $\left(\mathrm{q}_{\mathrm{a}}\right)$; y ocasionadas por diferencias en los tiempos de carga y descarga, la cantidad de traslados sin carga comúnmente llamado "movimientos de vacío", la cantidad de tiempos muertos, la distancia de recorrido en nuevos destinos o a la velocidad comercial. De esta forma, resulta importante una metodología que, de forma prospectiva, permita calcular los elementos del viaje, aún sin estadísticas previas, como el método del análisis del ciclo vehicular.

\subsection{Análisis del Ciclo Vehicular}

Una alternativa, para contemplar las variables del viaje y determinar de forma prospectiva la cantidad de producción $\left(\mathrm{q}_{\mathrm{a}}\right)$, es el método del Análisis del Ciclo Vehicular (ACV). El método del ACV es una forma sistemática para estudiar las operaciones de cualquier modo de transporte -incluyendo vehículo, contenedor o remolque- y analizar los tiempos y desplazamientos combinados con factores económicos, entre el momento en que se inicia un servicio y el instante en que se termina y se encuentra listo para reiniciar el servicio (De Buen y Almeida, 1992).

El ACV tiene tres componentes o ciclos, determinados por periodos de tiempo, las actividades y la distancia que recorren en los servicios (Manheim, 1979). El primero, es el ciclo operativo (CO), que es un periodo de tiempo continuo que inicia cuando se asigna el vehículo para realizar el servicio de transporte y termina después de que se entregan las mercancías, cuando el vehículo ingresa a la base y se encuentra listo para reiniciar el ciclo. En el ciclo operativo se registran todos los tiempos y los desplazamientos que los vehículos realizan -incluyendo los tiempos muertos y el tiempo de encierro o pernocta- cada uno asociado a la distancia que recorre. Así, el total del Tiempo del ciclo operativo $\left(\mathrm{T}_{\mathrm{co}}\right)$ y su Distancia $\left(\mathrm{D}_{\mathrm{co}}\right)$ se calculan de la siguiente forma:

$$
\begin{aligned}
& T_{c o}=\sum_{i=1}^{n} t_{i} \\
& D_{c o}=\sum_{i=1}^{n} d_{i}
\end{aligned}
$$

Donde $\mathrm{t}$ corresponde a los tiempos y d a las distancias de todas las actividades i que realiza el vehículo. Se incluyen los traslados con o sin mercancías, el tiempo de carga y descarga, los periodos de espera, el tiempo de que permanece estacionado o en encierro y, en general, todo el tiempo que transcurre con sus respectivas distancias, desde el inicio hasta el final del ciclo. 
El segundo es el Ciclo de Servicio (CS). Corresponde al tiempo en el que se realizan cierto número de ciclos operativos $\left(\mathrm{n}_{\mathrm{co}}\right)$, y se le suma el tiempo en el que el vehículo recibe mantenimiento programado, periodo conocido como Tiempo de servicio $\left(\mathrm{T}_{\mathrm{s}}\right)$. El tiempo del ciclo de servicio $\left(\mathrm{T}_{\mathrm{cs}}\right)$ se obtiene con la siguiente ecuación:

$$
\mathrm{T}_{\mathrm{cs}}=\mathrm{T}_{\mathrm{s}}+\mathrm{n}_{\mathrm{co}} \cdot \mathrm{T}_{\mathrm{co}}
$$

El número de ciclos operativos por ciclo de servicio $\left(\mathrm{n}_{\mathrm{co}}\right)$ se calcula dividiendo el rendimiento del mantenimiento -usualmente dado en distancia o tiempo- entre el consumo por ciclo operativo. Por ejemplo, para el caso donde se especifica que el mantenimiento se tiene que realizar cada vez que el vehículo cumple una determinada distancia de recorrido $\left(\mathrm{d}_{\mathrm{m}}\right)$, para obtener los ciclos operativos por ciclo de servicio $\left(\mathrm{n}_{\mathrm{co}}\right)$ sólo se divide la distancia de mantenimiento $\mathrm{d}_{\mathrm{m}}$ entre la distancia del ciclo operativo $\left(\mathrm{D}_{\mathrm{co}}\right)$.

$$
\mathrm{n}_{\mathrm{co}}=\mathrm{d}_{\mathrm{m}} / \mathrm{D}_{\text {co }}
$$

No obstante, es común que en México las empresas de transporte de carga por carretera realicen el mantenimiento de los vehículos durante los periodos de encierro o días no laborales, de tal forma que el Tiempo de servicio se considera igual a cero $\left(\mathrm{T}_{\mathrm{s}}=0\right)$. Además, cabe señalar que el número de ciclos operativos $\left(\mathrm{n}_{\mathrm{co}}\right)$ se determina con base a las necesidades de los vehículos y recomendaciones de los fabricantes que, usualmente, incluye periodos de tiempo o de distancia recorrida. En el transporte de carga por carretera es habitual que los fabricantes recomienden el mantenimiento después de cierto número de kilómetros, por lo que el número de ciclos operativos que se llevan a cabo en cada ciclo de servicio se obtiene al dividir el número de kilómetros recomendados para su mantenimiento entre la distancia que se recorren en cada ciclo operativo $\left(D_{c o}\right)$.

El tercer componente es el Ciclo Anual (CA). Como su nombre lo indica la duración es de un año; aunque se deben considerar sólo los días efectivamente trabajados, ya que hay empresas que no realizan actividades ciertos días por semana -tiempo no laborado $\left(\mathrm{t}_{\mathrm{la}}{ }^{-}\right.$- como los domingos o los periodos vacacionales. Así, el número de ciclos de servicio por año $\left(\mathrm{n}_{\mathrm{cs}}\right)$ se calcula a través del cociente formado entre el tiempo laborado por año $\left(\mathrm{T}_{\mathrm{la}}\right)$ y el tiempo del ciclo de servicio $\left(\mathrm{T}_{\mathrm{cs}}\right)$, tal y como se muestra en la siguiente ecuación:

$$
n_{c s}=\frac{T_{l a}}{T_{c s}}=\frac{365 \text { días }-t_{\overline{l a}}}{T_{c s}+n_{c o} \cdot T_{c o}}
$$

Con el número de ciclos operativos por año $\left(\mathrm{n}_{\mathrm{ca}}\right)$ se puede obtener el nivel de producción anual $\mathrm{q}_{\mathrm{n}}$. Por ejemplo, dado que se conoce la distancia por ciclo operativo, se puede obtener la cantidad de kilómetros recorridos por año y, con esto, el consumo de combustible, las emisiones contaminantes, los costos u otros datos de los que se tengan rendimientos y que pueden resultar de interés. El numero anual de ciclos operativos $\left(\mathrm{n}_{\mathrm{ca}}\right)$ se puede obtener multiplicando los ciclos operativos por ciclo de servicio $\left(\mathrm{n}_{\mathrm{co}}\right)$ y el número de ciclos de servicio por año $\left(\mathrm{n}_{\mathrm{cs}}\right)$, como se muestra en la siguiente formula:

$$
\mathrm{n}_{\mathrm{ca}}=\mathrm{n}_{\mathrm{co}} \cdot \mathrm{n}_{\mathrm{cs}}
$$

Finalmente, para conocer la estructura económica, el método del ACV contempla la recolección de los costos fijos y los costos variables. Usualmente se elaboran tres tablas, 1) la correspondiente a componentes de los ciclos operativos, de servicio y anual; 2) la que incluye los costos fijos; y 3) la correspondiente a los costos variables. En cada una se incluye suficiente nivel de detalle, como los rendimientos y los costos unitarios, para poder establecer, según se requiera, el costo de manera anual o por unidad de producción $\left(\mathrm{q}_{\mathrm{i}}\right)$. Asimismo, se puede agregar un porcentaje de utilidad, como lo muestra la ecuación 3 , para poder examinar los ingresos, los costos, la utilidad o el punto de equilibrio.

\section{Metodología}

El estudio se realizó en una empresa de manufactura que domina el mercado mexicano de sistemas construcción ligera en la que se requería evaluar los costos que generan las empresas subcontratadas de transporte de carga por carretera, para tener una forma objetiva y responsable de llevar a cabo las negociaciones de las tarifas y sus incrementos anuales.

La empresa embarcadora genera cerca de trece mil embarques por año, lo que le otorga cierto control y poder de negociación ante los transportistas. Sin embargo, en la empresa se contempla la importancia del desarrollo de proveedores de transporte y el valor que tiene su experiencia para evitar mermas en el manejo del producto, ya que los nuevos transportistas tienen una curva de aprendizaje que representa un alto costo para la empresa. Así que, la empresa embarcadora pretende ser justa en las tarifas y realizar esfuerzos para mejorar el desempeño de los proveedores de transporte y lograr estabilidad y rendimientos a largo plazo, no sólo en aspectos económicos sino también en los sociales y los ambientales.

Los envíos, tienen origen en tres plantas ubicadas en occidente, norte y oriente del país, y se realizan a toda la República Mexicana con 52 empresas de transporte por carretera de diferentes tamaños: $51.92 \%$ son microempresas (owner-operator u hombre-camión); $11.54 \%$ son conglomerados de Hombre-Camión, pero administradas por un agente o bróker; $13.46 \%$ son consideradas medianas (tienen entre 31 y 100 vehículos); y 3.85\% como grandes (con más de 100 vehículos). Asimismo, los embarques se llevan a cabo en la modalidad de carros completos (full truck load) tipo plataforma (flatbed truck) a través de camiones unitarios de tres ejes C3 (en proporción de 18.4\%), articulados con un sólo remolque T3S3 (77.4\%), y doblemente articulados T3S2R4 (4.5\%). 
En total, los transportistas utilizaron 492 vehículos tractivos para las necesidades de la empresa embarcadora. De esta forma, dada la diversidad de marcas, modelos y precios de vehículos, remolques, neumáticos, gastos de administración $\mathrm{y}$, en general, todos los costos directos e indirectos; durante abril de 2019, se realizó una encuesta en la que participaron todos los transportistas, para obtener los componentes de los costos fijos y los costos variables, incluyendo rendimientos o temporalidad de los gastos. Además, para tener parámetros de referencia, se solicitaron cotizaciones, y se complementaron con consultas electrónicas de los factores del costo.

En cuanto a los tiempos y desplazamientos correspondientes al ciclo operativo, la empresa embarcadora dispone de un sistema informático, llamado SISEMB (Sistema de Embarques), programado como parte de un sistema de ERP (Enterprise Resource Planning) en el que se registra el seguimiento a los embarques. Se incluyen datos como el horario en que el conductor llega a las instalaciones y se encuentra disponible para cargar, la hora en que ingresa al andén, la hora de inicio y término del proceso de carga y el horario de salida en el que se inició el viaje al destino final.

En todos estos procesos, los empleados del área de transporte y vigilancia utilizan computadoras para registran las fechas y horarios a través de casillas de verificación, que no pueden ser manipuladas por los usuarios y donde los horarios son capturados en tiempo real. Los datos se complementan con la información de horarios de arribo y descarga, que se obtienen a través de información que los clientes establecen en la documentación de entrega (notas de remisión) y de los reportes que los transportistas realizan a través sistemas de posicionamiento global -si disponen del servicio- o de reportes telefónicos que realizan los conductores.

De esta forma, se analizaron los registros correspondientes a los últimos doce meses sobre la duración y el tiempo de cada actividad $\mathrm{y}$, dado que se comportaron con una distribución normal (con 99.5\% de certeza), se estimaron los tiempos esperados para cada una de las actividades de los embarques.

Finalmente, utilizando los costos, junto con los tiempos y desplazamientos, se aplicó el método del Análisis del Ciclo Vehicular; se sistematizó el cálculo para nuevos destinos; y se realizó un análisis de sensibilidad de costos para cambios en la cantidad de producción, el precio del combustible, el costo de los peajes, o el factor de ajuste por viaje sin carga, entre otras variables.

\section{Resultados}

De las cincuenta y dos empresas de transporte que participaron en el sondeo, se determinaron los costos involucrados en el servicio de transporte de carga por carretera. En la tabla 1 se muestra el promedio de los costos fijos para un vehículo articulado. Cabe señalar que, la edad de la flota vehicular es de siete años; trece años para las empresas que dan servicio en la planta ubicada en el centrooriente del país y cinco años para la planta ubicada en el noreste. Dado que las empresas regularmente no tienen establecidas políticas de reemplazo de sus vehículos, se fijó en doce años el periodo de utilización, ya que esta etapa corresponde a una de las fases de reconstrucción vehicular y a uno de los tres periodos prioritarios para la reposición de los vehículos (Rafael, 2004).

\begin{tabular}{|c|c|c|c|c|}
\hline \multirow{21}{*}{$\begin{array}{l}\text { Tabla } 1 \text { Costos fijos } \\
\text { promedio en el transporte } \\
\text { carretero de sistemas de } \\
\text { construcción ligera }\end{array}$} & Concepto & $\begin{array}{c}\text { Costo } \\
\text { unitario }\end{array}$ & \multirow[t]{2}{*}{$\begin{array}{c}\text { Rendimiento } \\
\text { (años) }\end{array}$} & $\begin{array}{l}\text { Costo anual } \\
\text { (US\$) }\end{array}$ \\
\hline & Valor del vehículo descontando valor de recuperación & & & \\
\hline & Unidad motriz & $\$ 63,000.00$ & 12 & $\$ 5,250.00$ \\
\hline & Unidad de arrastre & $\$ 14,634.39$ & 12 & $\$ 1,219.53$ \\
\hline & Seguro & & & \\
\hline & Unidad motriz & $2,772.00$ & 1 & $\$ 2,772.00$ \\
\hline & Unidad de arrastre & 809.48 & 1 & $\$ \quad 809.48$ \\
\hline & Tasa de interés & & & \\
\hline & Unidad motriz & $6.00 \%$ & 1 & $\$ 3,780.00$ \\
\hline & Unidad de arrastre & $6.00 \%$ & 1 & $\$ 878.06$ \\
\hline & Expedición de permisos e imposiciones del vehículo & & & \\
\hline & Unidad motriz & 219.76 & 1 & 219.76 \\
\hline & Unidad de arrastre & 167.75 & 1 & 167.75 \\
\hline & Verificación de condiciones físico-mecánicas & & & \\
\hline & Unidad motriz & 62.20 & 0.5 & 124.39 \\
\hline & Unidad de arrastre & 54.42 & 1 & 54.42 \\
\hline & Verificación de emisión de contaminantes & & & \\
\hline & Unidad motriz & 22.70 & 0.5 & 45.40 \\
\hline & Gastos administrativos & $3,485.09$ & 1 & $\$ 3,485.09$ \\
\hline & Comunicaciones y GPS & 402.13 & 1 & $\$ \quad 402.13$ \\
\hline & Otros gastos indirectos & $2,680.84$ & 1 & $\$ 2,680.84$ \\
\hline
\end{tabular}


La renta de oficinas y talleres de mantenimiento por unidad, los pagos de servicios de luz, teléfono, agua, limpieza, vigilancia, los gastos en papelería, asesoría laboral, legal, seguros de las instalaciones y otros gastos, para efectos de presentación, fueron agrupados en dos rubros: los gastos administrativos y otros gastos indirectos. Estos datos dependen totalmente de cada empresa ya que, por ejemplo, las de tipo hombre-camión (Conductor dueño del vehículo) no tienen instalaciones administrativas; sólo las empresas grandes tienen talleres de mantenimiento y, sólo $68 \%$ de la flota vehicular tienen Sistemas de Posicionamiento Global (GPS).

La tabla 2, muestra el promedio de los costos variables para un vehículo articulado, su rendimiento y el costo que tienen en dólares por kilómetro (US\$/km). Aquí -además de los gastos derivados de la preparación de nuevos servicios, la limpieza de unidades, viáticos y peajes- se incluye los costos de operación de mayor importancia, tales como el combustible, el mantenimiento, los neumáticos y el salario del conductor. Cabe señalar que los pagos por concepto de seguridad social no son incluidos en los salarios puesto que estos fueron incluidos en los costos fijos; debido a que, en general, las empresas medianas y grandes le pagan una parte fija al conductor, cercana al salario mínimo, y el resto de su ingreso es a través en un porcentaje del costo del flete; que es de $8 \%$ para los conductores de los camiones unitarios, de $10 \%$ para vehículos articulados, y de $12 \%$ para los que llevan dos remolques.
Tabla 2 Costos Variables y sus rendimientos.

\begin{tabular}{|c|c|c|c|c|c|c|}
\hline Concepto & $\begin{array}{c}\text { Costo } \\
\text { unitario }\end{array}$ & Unidad & Rendimiento & Unidad & $\mathbf{U S \$ / ~ k m ~}$ & Costo por viaje \\
\hline Combustible & $\$ 1.14$ & $(\$ / \mathrm{Lt})$ & 2.2 & $(\mathrm{~km} / \mathrm{Lt})$ & $\$ 0.52$ & $\$ 333.98$ \\
\hline Neumáticos & $\$ 8,800.00$ & $\$ / 22$ pzas) & $70,000.0$ & (km/22pzas) & $\$ 0.13$ & $\$ 80.83$ \\
\hline Mantenimiento menor & $\$ 475.85$ & $(\$)$ & $15,432.0$ & $(\mathrm{~km})$ & $\$ 0.03$ & $\$ 19.83$ \\
\hline Mantenimiento mayor & $\$ 2,345.73$ & $(\$)$ & $200,000.0$ & $(\mathrm{~km})$ & $\$ 0.01$ & $\$ 7.54$ \\
\hline Salario conductor & $\$ 0.12$ & $(\$ / \mathrm{km})$ & 1.0 & $(\mathrm{~km})$ & $\$ 0.12$ & $\$ 77.93$ \\
\hline Otros gastos variables & $\$ 0.03$ & $(\$ / \mathrm{km})$ & 1.0 & $(\mathrm{~km})$ & $\$ 0.03$ & $\$ 21.55$ \\
\hline Peajes & $\$ 0.16$ & $(\$ / \mathrm{km})$ & 1.0 & $(\mathrm{~km})$ & $\$ 0.16$ & $\$ 102.22$ \\
\hline
\end{tabular}

Costo Variable Total $\mathrm{CV}_{\mathrm{km}}=1.00 \mathrm{CV}_{643 \cdot \mathrm{km}}=643.88$
Así, de los 14,823 embarques que se realizaron en el año de estudio, se tomaron todos los pares origen-destinos y se calculó el promedio de kilómetros recorridos $(613 \mathrm{~km}$ por viaje más 30 de posicionamiento y holgura) que fueron utilizados como base para el Análisis del Ciclo Vehicular. Con esto se obtuvo que $51.7 \%$ del tiempo los vehículos estuvieron sin movimiento -es decir, en maniobras de carga y descarga, estacionados, en pernocta o en mantenimiento$\mathrm{y}$; en cuanto a los costos, $84.4 \%$ corresponden a los costos variables, siendo el combustible el de mayor porcentaje $(43.8 \%)$, seguido de los peajes (13.4\%), los neumáticos $(10.6 \%)$ y la remuneración de los choferes $(10.2 \%)$.

En cuanto al análisis del ciclo operativo (tabla 3), los transportistas tardan 2.85 horas en posicionar un vehículo en el área de carga de la empresa embarcadora (actividades 1 a 6). Ahí, la estancia del vehículo es de 6.84 horas durante los procesos de carga, documentación, sujeción de la carga y cubrir las mercancías con una lona, dado que son remolques de tipo plataforma (actividades 7 a 11). Luego el vehículo realiza el recorrido hasta el destinatario (actividad 12).
En promedio, se tienen una velocidad comercial de 35 kilómetros por hora, en la que se incluyen paradas, tiempo de descanso del chofer, tiempos de holgura y demás demoras. Ya con el destinatario, el promedio es de cuatro horas en la descarga (actividad 13); luego el transportista se dirige hacia la base o pensión, y junto con el encierro o pernocta (actividades 14 y 15) suman nueve horas. En total, se juntan 13.69 horas en el Tiempo de posicionamiento y maniobras de carga y descarga $\left(\mathrm{T}_{\mathrm{pm}}\right)$ y nueve como holgura para iniciar nuevos servicios $\left(\mathrm{T}_{\mathrm{h}}\right)$.

En la tabla 2, se tiene que el mantenimiento preventivo $\left(d_{m}\right)$ se realiza en promedio cada 15,432 kilómetros y, en la tabla 3, se muestra que el tiempo por ciclo operativo $\left(\mathrm{T}_{\mathrm{co}}\right)$ es de 39.2 horas (1.6 días); mientras que la distancia de recorrido $\left(\mathrm{D}_{\mathrm{co}}\right)$ es de 643 kilómetros. Si se considera que sólo se trabajan 300 días por año $\left(\mathrm{T}_{\mathrm{la}}=300\right.$ días) y que el mantenimiento se realiza durante los días no laborales, es decir $\mathrm{T}_{\mathrm{s}}=0$, con las ecuaciones 8,9 y 10 se obtiene que: $\mathrm{n}_{\mathrm{co}}=24$ ciclos operativos por ciclo de servicio, $\mathrm{T}_{\mathrm{cs}}=39.2$ días $\mathrm{y}, \mathrm{n}_{\mathrm{cs}}=183.6$ ciclos operativos por año. 
Tabla 3 Ciclo operativo

\begin{tabular}{|c|c|c|c|}
\hline i & Actividad & $\begin{array}{c}\mathbf{t}_{\mathbf{i}} \\
\text { (horas) }\end{array}$ & $\begin{array}{c}D_{i} \\
(\mathbf{k m})\end{array}$ \\
\hline 1 & Conductor arriba a instalaciones del transportista. & 0.08 & 0 \\
\hline 2 & Reabastecimiento y preparación para nuevos servicios & 0.50 & 0 \\
\hline 3 & Conductor recibe instrucciones. & 0.17 & 0 \\
\hline 4 & Conductor revisa vehículo y engancha remolque. & 0.50 & 0 \\
\hline 5 & Conductor recibe documentos. & 0.17 & 0 \\
\hline 6 & Recorrido 1 (de las instalaciones del transportista a las del embarcador). & 0.43 & 15 \\
\hline 7 & Conductor registra su llegada y espera posicionamiento de anden. & 1.00 & 0 \\
\hline 8 & Conductor realiza maniobras de posicionamiento en anden. & 0.17 & 0 \\
\hline 9 & Las mercancías se cargan del remolque. & 3.50 & 0 \\
\hline 10 & Se coloca la lona para protección de la carga (Plataforma) & 2.00 & 0 \\
\hline 11 & Entrega de documentos y salida de área de embarques. & 0.17 & 0 \\
\hline 12 & Recorrido 2 (Del embarcador al destinatario). & 17.51 & 613 \\
\hline 13 & $\begin{array}{l}\text { Las mercancías llegan al destino, el conductor se registra, espera turno y } \\
\text { tiempo de descarga. }\end{array}$ & 4.00 & 0 \\
\hline 14 & Recorrido 3 (Destinatario a pensión). & 1.00 & 15 \\
\hline 15 & Pernocta y tiempo de encierro. & 8.00 & 0 \\
\hline & $\begin{array}{ll}\text { Total } \\
\end{array}$ & $\Gamma c 0=39.21$ & $\operatorname{co}=643$ \\
\hline
\end{tabular}

Como se indicó anteriormente, el número de ciclos operativos (viajes) puede ser considerado el nivel de producción $\left(\mathrm{q}_{\mathrm{n}}\right) \mathrm{o}$, en su caso, puede ser utilizado para proyectar otras unidades de medida -como los kilómetros o el combustible anual- multiplicando los ciclos operativos por año $\left(\mathrm{n}_{\mathrm{cs}}\right)$ por el consumo por viaje. Dado que por cada ciclo operativo (viaje) se recorren 643 kilómetros ( $\left.\mathrm{D}_{c o}\right)$ y se proyectan 183.6 ciclos operativos por año $\left(\mathrm{n}_{\mathrm{cs}}\right)$, de la multiplicación se obtienen 118,083 km-año.

De esta forma, para establecer la tarifa de transporte por unidad de producción $\left[\mathrm{F}\left(\mathrm{q}_{\mathrm{i}}\right)\right]$, primero se calcularon los costos fijos correspondientes al ciclo operativo, dividiendo el costo fijo anual entre el número de ciclos operativos por año $\left(\mathrm{CF}_{\mathrm{i}}=\mathrm{CF}_{\mathrm{a}} / \mathrm{n}_{\mathrm{cs}}=\$ 119.19\right.$ por ciclo o $\$ 0.19$ por kilómetro); y los costos variables, multiplicando el costo de cada kilómetro por la distancia del ciclo operativo $\left[C V_{i}\left(q_{i}\right)=C_{k m} * D_{c o}=\$ 643.88\right]$. Posteriormente, utilizando la ecuación 3 y un porcentaje de utilidad $(\% \mathrm{U})$ de $13.04 \%$, de acuerdo con el promedio reportado, se obtiene una tarifa $\left[\mathrm{F}\left(\mathrm{q}_{\mathrm{i}}\right)\right]$ de US\$877.53 por viaje; y un punto de equilibrio ubicado en 93.7 ciclos operativos (ecuación 5), lo que indica que la empresa transportista sólo comenzará a tener utilidad positiva a partir del viaje número 94.

Dado que el análisis del ciclo vehicular depende de los rendimientos de los diferentes factores que intervienen en el costo, y estos cambian de acuerdo a las condiciones específicas como la sinuosidad del camino, las pendientes, la rigurosidad del mantenimiento, la antigüedad y utilización del vehículo; se realizó un análisis de sensibilidad para establecer en qué medida, una vez que se ha acordado una tarifa, la generalización o la utilización de los promedios podría afectar los costos y, por tanto, a la utilidad.

\subsection{Análisis de sensibilidad}

Con el análisis de sensibilidad se pueden observar los elementos que más influyen en el costo total (CT). Para calcular la variación $(\Delta \mathrm{CT})$ basta con multiplicar el incremento porcentual de alguno de los factores del costo $\left(\Delta \mathrm{C}_{\mathrm{i}}\right)$ por la proporción que el elemento representa en relación con el costo total inicial $\left(\mathrm{C}_{\mathrm{i}} / \mathrm{CT}\right)$. Para analizar los costos por ciclo operativo (co), se puede utilizar la siguiente ecuación:

$$
\Delta C T_{c o}=\Delta C_{i \cdot c o} \cdot \frac{C_{i \cdot c o}}{C T_{c o}}
$$

Así, por ejemplo, el costo del diésel por ciclo operativo $\left(\mathrm{C}_{\text {désel•co }}=\$ 333.98\right)$ representa 0.438 de los costos totales $\left(\mathrm{CT}_{\mathrm{co}}=\$ 763.07\right)$ por lo que un incremento de $20 \%$ en el costo del combustible $\left(\Delta \mathrm{C}_{\text {diésel }}=0.20\right)$ generaría un incremento de $8.75 \%$, de acuerdo a los siguientes cálculos en $\Delta \mathrm{CT}_{\mathrm{co}}=0.20 *$ $\$ 333.98 \$ 763.07$.

La sumatoria de todos los incrementos relativos de los elementos del costo $\left(\sum \Delta \mathrm{C}_{\mathrm{i}}\right)$ pueden ser útiles para calcular la inflación real del costo de servicio de transporte. Sin embargo, para efectos del análisis de sensibilidad, se consideró un intervalo con decrementos e incrementos de hasta 0.50 en los costos $[-50 \%, 50 \%]$. En la figura 1, se muestran la variación de los elementos que más impactan en el costo total; y no sólo se incluyeron los costos unitarios o costo por ciclo operativo, sino que se analizó el tiempo y el rendimiento de cada una de las actividades del ciclo operativo. 
Como es de esperarse, el combustible, al tener la mayor proporción en la estructura de costos $(43.8 \%)$, es el elemento que más impacta en el costo final $\mathrm{y}$, tanto el rendimiento como el costo unitario se relacionan directamente con el costo total. Así, por ejemplo, si el rendimiento de combustible de alguna de las empresas es 50\% menor al promedio considerado (es decir cambia de 2.2 a $1.1 \mathrm{~km} / \mathrm{Lt}$ ) el incremento en el costo total sería de $43.77 \%$, llegando a ser $60.9 \%$ en relación con el total. Mientras que un incremento de $50 \%$ en el costo del combustible (de \$1.14 a \$1.71 por Lt) representaría un aumento de $21.9 \%$ en el costo total. En este sentido, un incremento en el rendimiento de 50\% -es decir, pasar de un vehículo de 2.2 a otro de $3.3 \mathrm{~km} / \mathrm{Lt}$ - generaría una disminución de $14.6 \%$ de los costos totales.

Por supuesto, el consumo de combustible también tiene una fuerte relación con la distancia de recorrido. Aumentar en $50 \%$ los kilómetros por viaje incrementan $40.2 \%$ el costo total, por lo que el costo marginal disminuye sólo alrededor de $5.3 \%$. Por lo que este factor toma relevancia en los costos al aumentar los kilómetros recorridos sin carga.
Figura 1 Impacto

porcentual en costo total por variación en elementos del costo por ciclo operativo
Figura 2 Variación en los elementos del costo con menor impacto en el costo total por ciclo operativo
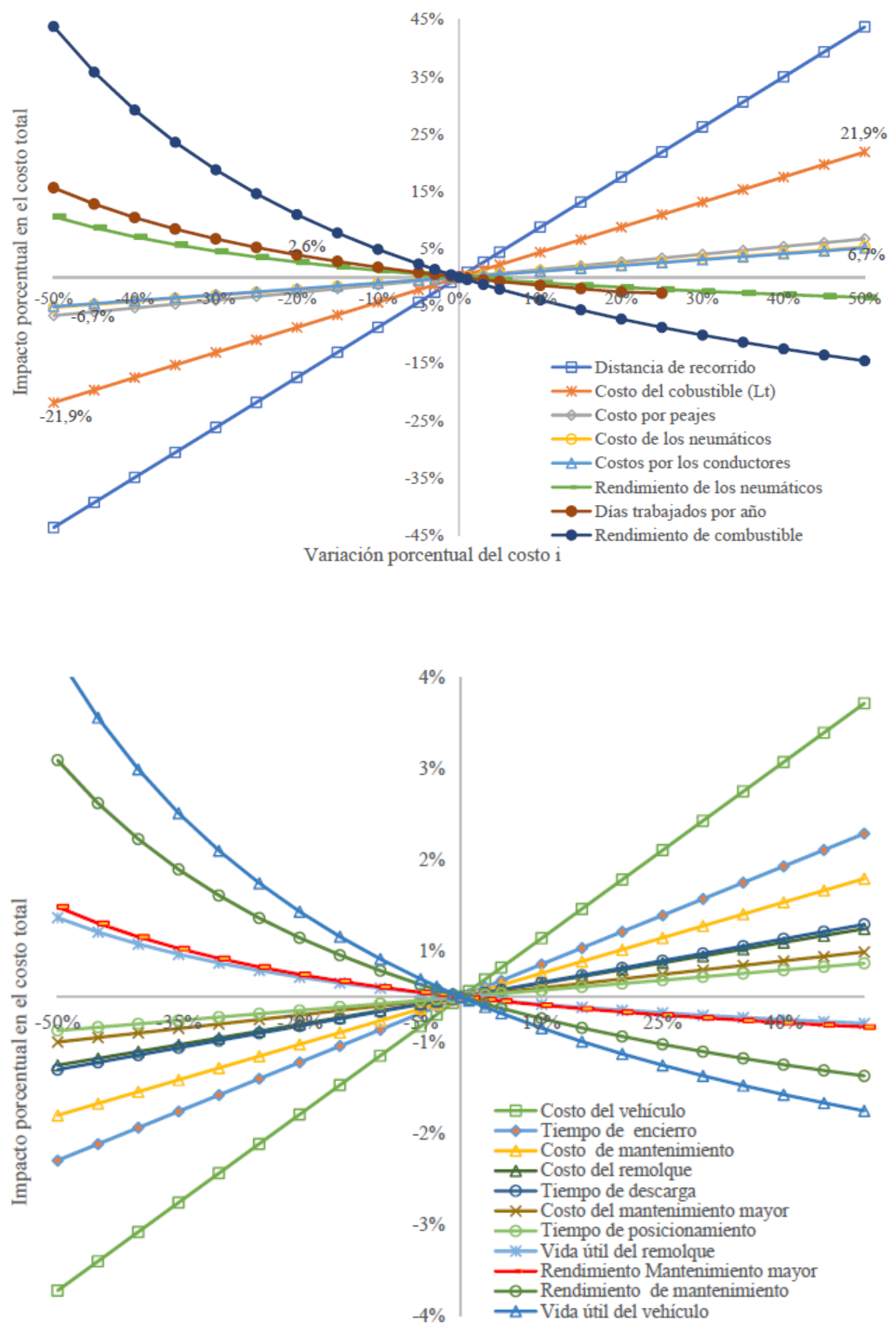

Variación porcentual del costo $\mathrm{i}$ 
Asimismo, le siguen los costos de los peajes, los costos de los neumáticos, los gastos en salarios de los conductores $\mathrm{y}$, en menor medida, los que se relacionan a los costos fijos, como la vida útil del vehículo y costos ocasionados por partes en el proceso, y su tiempo, donde los vehículos permanecen sin movimiento -como el tiempo de encierro, el tiempo que tardan en cargar las mercancías o el tiempo de descarga-. Por ejemplo, en la figura 2 , se puede observar que con un cambio de $\pm 50 \%$ en la vida útil del vehículo -es decir, que cambia de doce a seis o dieciocho años- respectivamente incrementa $3.8 \%$ y decrece $1.3 \%$ en los costos totales. Otros impactos por cambios en los costos, tiempos del ciclo operativo o los rendimientos, también se muestran en la figura 2.

Finalmente, es importante mencionar que, en cuanto a los días trabajados por año, si la empresa tuviera operaciones los 365 días del año, 21.6\% más, se reducirían cerca de 3.05\% el costo marginal; mientras que, si trabaja $22 \%$ menos días, el costo aumenta alrededor de $4.1 \%$. Sin embargo, aunque parecen ser cantidades que aportan poco en cuanto a los costos, en términos de utilidad resultan muy relevante debido a que se incrementa el número de ciclos operativos por año (a 223 ) y, por tanto, se incrementa hasta $\pm 18.3 \%$ la utilidad total anual.

\section{Discusión y conclusiones}

La mayor limitación del método del ACV se presenta cuando las rutas son dinámicas. En estos casos los transportistas llegan a su punto final y su nuevo destino es diferente al origen inicial. Incluso, en muchos casos, los transportistas no tienen negociado un viaje de regreso y no saben dónde y cuándo cargaran; dicho de otra forma, no saben cuál será su nuevo destino. Esto ocurre de manera habitual en México puesto que los pares origen-destino de los intercambios comerciales no son simétricos y no se tiene carga para regresar al punto de origen. Ante esta situación, los transportistas llevan a cabo algunas de las siguientes estrategias: 1) se desplazan a alguna ciudad cercana y de ahí vuelven a carga para dirigirse al punto de partida; 2) se regresan sin carga hasta el punto de origen inicial o; 3) tiene que esperar uno o varios días hasta que haya carga disponible.

Para el primer caso, cuando es una actividad habitual, basta con modificar el tiempo y la distancia del destinatario al nuevo punto de carga en el ciclo operativo, actividades correspondientes a $\mathrm{t}_{14} \mathrm{y} \mathrm{d}_{14}$ en la tabla 3 . De esta forma, por ejemplo, desplazarse 100 kilómetros hacia un nuevo cliente $\left(\mathrm{d}_{14}=100 \mathrm{~km}\right)$ generaría un incremento en el costo cercano a $11.2 \%$. El segundo caso, prácticamente, consiste en duplicar el tiempo y la distancia del embarcador al destinatario ( $\mathrm{t}_{12} \mathrm{y} \mathrm{d}_{12}$ en la tabla 3$)$; lo que incrementa fuertemente la tarifa al cliente (87.4\%). Mientras que en el caso 3, modificar el tiempo de encierro $t_{15}$, ocasiona cerca de $9.5 \%$ adicional por cada 24 horas de espera. En suma, cuando los desplazamientos no son habituales, el ACV se debe combinar con registros históricos de las empresas (estadísticas) para aumentar la potencia y la exactitud de los cálculos.

No obstante, el Análisis del Ciclo Vehicular (ACV) es una herramienta que facilita el conocimiento de los costos relacionados a las operaciones de los vehículos y, por tanto, permite establecer precios con relativa facilidad. El método del ACV puede ser utilizado tanto para empresas grandes como para empresas de tipo hombre camión; y no requiere esfuerzos de programación, ya que puede ser computado a través de una hoja de cálculo. Asimismo, puede ser empleado por cualquiera de los agentes implicados en el movimiento de mercancías -embarcador, porteador o destinatario- y ser utilizado durante la contratación o negociación de tarifas y, en general, para tomar decisiones con pleno conocimiento de los costos y las operaciones de los vehículos.

Por su parte, el análisis de sensibilidad permite identificar el impacto de los elementos del costo en el precio final. En este sentido, llama la atención que ni el rendimiento de los neumáticos, ni el costo de los conductores tienen un fuerte impacto en el costo. A pesar de la diferencia en el desgaste de los neumáticos entre los vehículos que circulan bajo diferentes características -como transitar en carreteras del norte y sur del país, el estado general de la carpeta de rodamiento, la sinuosidad, las elevaciones o las pendientes de la sierra- una variación de $-50 \%$ en el rendimiento ocasiona $10.6 \%$ de incremento en el costo. Asimismo, aumentar la misma proporción el gasto en los conductores sólo afecta en $5.1 \%$ el costo final. Se recomienda explorar cómo al mejorar la situación de los conductores -no sólo el salario, sino las condiciones laborales en general- puede ayudar a disminuir la escasez de conductores, que es una situación nacional (Berrones-Sanz, 2020), al mismo tiempo que se mejora el nivel de servicio y la competitividad.

La variación del costo de mantenimiento tampoco muestra un impacto significativo $\mathrm{y}$, aunque no se considera el incremento longitudinal, a corto plazo no representa un incremento relevante $(1.3 \%)$. Perceptiblemente, el costo del mantenimiento aumenta con la antigüedad del vehículo, por lo que se deben considerar los periodos prioritarios para la reposición de los vehículos y el periodo óptimo de renovación; que para una empresa con suficiente disponibilidad financiera es de entre cinco y siete años (Rafael, 2004). Asimismo, los vehículos más antiguos no realizan el mantenimiento preventivo con la frecuencia adecuada $\mathrm{y}$, generalmente, optan por el mantenimiento correctivo. Es así como se infiere que, para no alejarse de una zona que resulta cómoda, las empresas hombres-camión se enfocan a servicios con distancias más cortas y conectadas a cadenas productivas locales. 
Además, en cuanto al rendimiento de combustible de los vehículos antiguos, no sólo se afecta a la competitividad del sector, sino que se generan una serie de externalidades relacionadas a la emisión de gases contaminantes, desgaste de las carreteras y accidentes viales. En este sentido, los resultados empíricos muestran que los vehículos utilizados por la empresa tienen un rendimiento promedio de $2.2 \mathrm{~km} /$ Lt correspondiente a vehículos con siete años de antigüedad. Sin embargo, el promedio de edad de la flota vehicular para las empresas hombre-camión es de 13 años y, en los vehículos de mayor edad (con hasta 33 años de utilización) el rendimiento registrado fue de hasta $1 \mathrm{~km} / \mathrm{Lt}$; por lo que la diferencia del impacto en el costo total es muy relevante $(43.77 \%)$.

A pesar de lo portentoso de estos datos, la empresa embarcadora del caso de estudio utiliza menor proporción de empresas pequeñas $(30.8 \%)$ y de tipo hombre camión $(51.9 \%)$ que los registrados en el total nacional total nacional que es de $16.5 \%$ y $80.7 \%$ respectivamente (DGAF, 2018). Además, la flota vehicular, con promedio de 13 años, es menor que el promedio de la flota nacional de 17 años [t(499) $=-38.42, \mathrm{p}<.001]$.

Los transportistas de empresas pequeñas y de tipo hombrecamión tienen vehículos antiguos y sus administradores, generalmente, desconocen la estructura de costos, no contemplan el financiamiento y el reemplazo del vehículo $y$, con tal de mantenerse activos, pueden ofrecer tarifas muy atractivas para los embarcadores, que están hasta por debajo de los costos de operación. De esta forma, a pesar de las deficiencias en el servicio, los atrasos tecnológicos y las externalidades que generan este tipo de empresas, por conveniencia económica son contratadas de forma regular para transportas las mercancías.

Así, aunque los vehículos que se utilizan en las diferentes cadenas de suministro se seleccionan de acuerdo a las características tecnológicas de los vehículos y los requerimientos propios de cada producto; la antigüedad de la flota vehicular nacional y su desempeño, puede ser equiparado a los resultados empíricos, por lo que se destaca la necesidad de crear programas para renovar la flota vehicular nacional y, así, aumentar la productividad y la competitividad del transporte de carga por carretera en México.

Por su parte, en el caso de estudio, también se reveló que los costos fijos relacionados a la compra del vehículo pueden incrementar el costo por viaje hasta en 3.2\% al incrementar la inversión en 50\%. Por supuesto, esto depende de la estabilidad en el número de viajes generados por año. Asimismo, estos resultados están siendo utilizados para elaborar programas de sustitución de vehículos y, así, disminuir la edad de la flota vehicular, reducir el consumo de combustible, la generación de emisiones contaminantes, el deterioro de las carreteras, los accidentes de tránsito y, en general, contribuir en las tres dimensiones del desarrollo sostenible. De esta forma, el Análisis del Ciclo Vehicular (ACV) está siendo empleado para establecer tarifas que permitan la competitividad y la sostenibilidad financiera a largo plazo. Además, con la examinación individual de los proveedores, se pretende identificar y compartir las buenas prácticas en cuanto a la productividad, la eficiencia y los costos.

A pesar de que se habla que muchos elementos del costo no tienen un fuerte efecto en el total, se sabe que todos son importantes para lograr la competitividad; ya que la eficiencia acumulada puede traer importantes ahorros o, por el contrario, fuerte efectos negativos. En este sentido, el ACV también ha permitido identificar áreas de oportunidad en cuanto a la empresa embarcadora y los destinatarios (sus clientes). Por ejemplo, en cuanto al tiempo y los procesos analizados en el ciclo operativo se ha identificado que es importante que, aunque con menor impacto en los costos, se disminuyan los tiempos muertos, los tiempos de carga, los tiempos de descarga y, en general, todo el tiempo que los vehículos pasa sin movimiento. Evidentemente, un vehículo en cualquier modo de transporte será más rentable en medida que pase mayor tiempo en movimiento (con carga), por lo que debe lograrse optimizar cada uno de los rendimientos que intervienen en los insumos. Así, el Análisis del Ciclo Vehicular ha permitido crear indicadores como punto de partida para ejercer acciones que disminuyan el tiempo y hagan más eficientes los procesos de embarques.

Finalmente, se puede concluir que el método del ACV resulta económico práctico y sencillo, se puede implementar y sistematizar fácilmente a través de una hoja de cálculo en cualquier ordenador, y no requiere mucha capacitación. Por tal motivo resulta una herramienta esencial para todos los involucrados en el transporte, especialmente los transportistas y las personas que tienen que calcular y negociar tarifas con rapidez y simplicidad. 


\section{Referencias}

ALVEAR, Sandra and RODRÍGUEZ, Patricia C. (2006) 'Estimación del Costo por Kilómetro y de los Márgenes de una Empresa de Transporte de Carga, para la Industria Agrícola, Región del Maule, Chile', Panorama Socioeconómico 24(32): 48-57.

ARROYO, José Antonio, TORRES, Guillermo, GONZÁLEZ, José Alejandro and HERNÁNDEZ, Salvador (2016) Costos de operación base de los vehículos representativos del transporte interurbano 2016. Querétaro: Instituto Mexicano del Transporte.

BERRONES-SANZ, Luis David (2020) 'Road freight transport in Mexico: production and employment', Análisis Económico 35(90): 142-72.

CANACAR (2002) Referencias de costos mínimos para el autotransporte de carga general. México: Cámara Nacional del Autotransporte de Carga (CANACAR).

DE BUEN, Orcar and ALMEIDA, Alberto (1992) Análisis del ciclo vehicular. Querétaro: Instituto Mexicano del Transporte.

DE RUS, Ginés, CAMPOS, Javier and NOMBELA, Gustavo (2003) Economía del transporte. Barcelona: Antoni Bosch.

DGAF (2018) Estadística básica del Autotransporte Federal 2018. México: Dirección General de Autotransporte Federal (DGAF).

DONDO, Rodolfo G. and MENDEZ, Carlos A. (2014) 'A Branch-and-Price Approach To Manage Cargo Consolidation and Distribution in Supply Chains', Industrial \& Engineering Chemistry Research 53(44): 17226-39.

HOLGUÍN, José, WOJTOWICZ, Jeffrey, GONZÁLEZ, Carlos, LAWRENCE, Michael, SKOLNIK, Jonathan, BROOKS, Michael, ZHANG, Shanshan, STRAUSS, Anne and TAVASSZY, Lóri (2013) Freight Data Cost Elements. Washington D.C.: Transportation Research Board.

HOOPER, Alan and MURRAY, Dan (2019) An Analysis of the Operational Costs of Trucking: 2019 Update. Arlington, Va: American Transportation Research Institute.
IMT (2018) Sistema de Costos del Autotransporte de Mercancías (SI-CAM), Instituto Mexicano del Transporte, https://imt.mx/sicam/Bienvenida.html (consulted 27 Nov 2020).

IZADI, A., NABIPOUR, M. and TITIDEZH, O. (2020) 'Cost Models and Cost Factors of Road Freight Transportation: A Literature Review and Model Structure', Fuzzy Information and Engineering.

JIMÉNEZ, José Elías and JIMÉNEZ, Jocelyn (2016) Logística del autotransporte de carga: estrategias de gestión. Querétaro: Instituto Mexicano del Transporte.

LITMAN, Todd Alexander (2009) Transportation Cost and Benefit Analysis: Techniques, Estimates and Implications. Canada: Victoria Transport Policy Institute.

MANHEIM, Marvin L. (1979) Fundamentals of transportation systems analysis. Cambridge, MA, London: MIT Press.

MORENO QUINTERO, Eric (2014) Índices de Precios en el Transporte por Carretera. Querétaro: Instituto Mexicano del Transporte.

RAFAEL, Mercedes Yolanda (2004) Métodos para la renovación de vehículos de autotransporte de servicio pesado. Querétaro: Instituto Mexicano del Transporte.

RITTENBERG, Libby and TREGARTHEN, Timothy (2018) Principles of macroeconomics, 3rd edn. Boston, MA: FlatWorld Publishing.

SCHADE, W., DOLL, C., MAIBACH, M., PETER, M., CRESPO, F., CARVALHO, D., CAIADO, G., CONTI, M., LILICO, A. and AFRAZ, N. (2006) COMPETE Final Report: Analysis of the contribution of transport policies to the competitiveness of the EU economy and comparison with the United States. Karlsruhe, Germany: European Commission.

SCHWARZE, Silvia (2016) 'Pricing strategies for the sitedependent vehicle routing problem', OR Spectrum 38(1): 137-73.

SEQUERA, Nahir (2014) 'Subjectivity and Objectivity of value', Comunidad y Salud 12(1): 64-8. 
TOPALOGLU, Huseyin and POWELL, Warren (2007) 'Incorporating Pricing Decisions into the Stochastic Dynamic Fleet Management Problem', Transportation Science 41(3): 281-301.

TSAO, Yu-Chung, ZHANG, Qinhong and CHEN, TsungHui (2016) 'Multi-item distribution network design problems under volume discount on transportation cost', International Journal of Production Research 54(2): 426-43.

YI, Junmin, DONG, Yuanyuan, SHI, Ting and ZHOU, Jing (2007) 'A two-stage model of vehicle routing and transport service pricing with backhauls', in 2007 IEEE International Conference on Grey Systems and Intelligent Services, pp. 1168-72: IEEE.

ZIKOPOULOS, C. (2019) 'Determination of freight rates under stochastic demand and freight consolidation savings', International Journal of Production Research 57(17): 5556-73. 